\title{
Effects of phytohormones and position on mother stem on culm cutting of Thyrsostachys siamensis gamble
}

\author{
Cham V. Mac ${ }^{1 *}$, Ha V. H. La ${ }^{1}, \&$ Thang V. Giang ${ }^{2}$ \\ ${ }^{1}$ Faculty of Forestry, Nong Lam University, Ho Chi Minh City, Vietnam \\ ${ }^{2}$ Forestry Science and Technology Association, Ho Chi Minh City, Vietnam
}

ARTICLE INFO
Research Paper
Received: May 07, 2018
Revised: July 19, 2018
Accepted: August 13, 2018
Keywords
Culm cuttings
Phytohormones
Position on mother stem
Thyrsostachys siamensis Gamble Nam Bo

\section{${ }^{*}$ Corresponding author}

Mac Van Cham

Email: macvancham@hcmuaf.edu.vn

\begin{abstract}
The effects of phytohormones and positions on mother stem on shooting rate, number of shoots per cut, foot diameter of shoots, height of shoots, rooting rate, average number and length of roots by culm cuttings of Thyrsostachys siamensis Gamble Nam Bo were investigated. In this study, the phytohormones used were NAA, IBA and HVP. Cuttings were taken in three positions: near the root (V1), between the stem (V2) and near the tops (V3). The experiment was arranged randomly with 3 replications, with 36 culm cuttings per treatment. The results showed that the groups treated with NAA had highest shooting rate $(91.7 \%)$ and highest number of shoots per cut (3.39 shoots/cut). The phytohormones did not significantly affect the foot diameter of the shoots but the height of the shoots. The NAA gave highest rooting rate $(87.04 \%)$ and highest number of roots $(8.5$ roots/cuttings). The positions on mother stems did not significantly affect the shooting rate, but they significantly affected the number of shoots per cut. The foot diameter of the shoots, the height of the shoots, the rooting rate and the average root length of the cuttings taken between the stems were greater than those of near the root and near the top. In addition, the highest number of roots was observed when cuttings were taken at near the root.
\end{abstract}

Cited as: Mac, C. V., La, H. V. H., \& Giang, T. V. (2019). Effects of phytohormones and position on mother stem on culm cutting of Thyrsostachys siamensis gamble. The Journal of Agriculture and Development 18(2), 71-77. 


\title{
Ảnh hưởng của chất điều hòa sinh trưởng và vị trí lấy HOM đến khả năng giâm HOM thân tre Tầm vông (Thyrsostachys siamensis Gamble) Nam Bộ
}

\author{
Mạc Văn Chăm ${ }^{1 *}$, La Vĩnh Hải Hà ${ }^{1}$ \& Giang Văn Thắng ${ }^{2}$ \\ ${ }^{1}$ Khoa Lâm Nghiệp, Trường Đại Học Nông Lâm TP. Hồ Chí Minh, TP. Hồ Chí Minh \\ ${ }^{2}$ Hội Khoa Học Kỹ Thuật Lâm Nghiệp TP. Hồ Chí Minh, TP. Hồ Chí Minh
}

\section{THÔNG TIN BÀI BÁO}

\section{Bài báo khoa học}

Ngày nhận: 07/05/2018

Ngày chỉnh sửa: 19/07/2018

Ngày chấp nhận: 13/08/2018

\section{Từ khóa}

Chất điều hòa sinh trưởng

Giâm hom thân

Tre Tầm vông Nam Bộ

Vị trí lấy hom

*Tác giả liên hệ

Mạc Văn Chăm

Email: macvancham@hcmuaf.edu.vn

\section{TÓM TẮT}

Bài báo này giới thiệu kết quả nghiên cứu về ảnh hưởng của chất điều hòa sinh trưởng (ĐHST) và vị trí lấy hom đến khả năng nảy chồi, số lượng chồi trên mỗi hom, đường kính chân măng, chiều cao măng, tỷ lệ ra rễ, số lượng rễ và chiều dài rễ trung bình trên mỗi hom khi giâm hom thân tre Tầm vông Nam Bộ. Trong nghiên cứu này, các chất điều hòa sinh trưởng được sử dụng là NAA, IBA và HVP. Hom thân đem giâm được lấy ở 3 vị trí: gần gốc (V1), giữa thân (V2) và gần ngọn (V3). Thí nghiệm được bố trí theo kiểu hoàn toàn ngẫu nhiên với 3 lần lặp lại, mỗi nghiệm thức của thí nghiệm có 36 hom. Kết quả cho thấy, khi xử lý NAA cho tỷ lệ nảy chồi $(91,7 \%)$ và số lượng chồi trên mỗi hom $(3,39$ chồi/hom) là cao nhất. Các chất ĐHST ảnh hưởng không rõ rệt đến đường kính của chân măng nhưng ảnh hưởng rõ rệt đến chiều cao của măng. NAA là chất ĐHST cho tỷ lệ ra rễ $(87,04 \%)$ và số lượng rễ trung bình (8,5 rễ/hom) lớn nhất. Vị trí lấy hom ảnh hưởng không rõ rệt đến tỷ lệ nảy chồi nhưng ảnh hưởng rất rõ rệt đến số lượng chồi trung bình trên mỗi hom thân tre Tầm vông Nam Bộ. Đường kính chân măng, chiều cao trung bình của măng, tỷ lệ ra rễ và chiều dài rễ trung bình của hom lấy ở vị trí giữa thân lớn hơn 2 vị trí còn lại. Bên cạnh đó, số lượng rễ trung bình cao nhất khi lấy hom ở vị trí gần gốc.

\section{1. Đặt Vấn Đề}

Tầm vông (Thyrsostachys siamensis Gamble) là một loài trong họ Tre trúc, phân bố tự nhiên rộng rãi ở dạng rừng thuần loài tại Myanmar, Thái Lan. Tầm vông đã được trồng ở nhiều nước trong khu vực. Ở Việt Nam, cây Tầm vông được trồng chủ yếu ở các tỉnh phía Nam. Tầm vông là loài tre trung bình, thân rất thẳng và đặc, dùng làm vật liệu xây dựng, làm nhà (rui mè) và các đồ dùng trong gia đình như làm cán cuốc, thuổng, dao và giáo mác (vì vậy có tên là cán giáo). Trong giao thông, tầm vông làm rào chống, sào căng buồm. Trong công nghiệp chế biến, tầm vông có thể làm nguyên liệu giấy hoặc làm thủ công mỹ nghệ (Nguyen, 2005).

Vai trò và công dụng của cây tre Tầm vông trong đời sống hằng ngày của chúng ta vô cùng đa dạng và hữu ích. Tuy nhiên, trong thời gian qua việc nghiên cứu và gây trồng loài cây này còn mang tính tự phát, chỉ dừng lại ở giâm hom gốc và chiết cành chưa đáp ứng được nhu cầu về giống của thực tiễn sản xuất. Trong khâu kỹ thuật nhân giống, ngoài yếu tố chọn tuổi cây mẹ thì vị trí lấy hom và loại chất điều hòa sinh trưởng thích hợp cũng cần được quan tâm nghiên cứu. Do đó, để nhân giống thành công thì bên cạnh đặc tính sinh học của loài cây, nó còn được ảnh hưởng bởi chất lượng của hom giống đem giâm mà cụ thể là ảnh hưởng của từng vị trí lấy hom trên thân cây tre Tầm vông mẹ và chất kích thích cho khả năng nảy chồi và ra rễ của hom giâm.

Xuất phát từ thực tiễn đó, để góp phần làm rõ sự ảnh hưởng của từng vị trí lấy hom và loại chất điều hòa sinh trưởng đến khả năng giâm hom thân tre Tầm vông Nam bộ, nghiên cứu này đã 
được thực hiện.

\section{Vật Liệu và Phương Pháp Nghiên Cứu}

\subsection{Vật liệu nghiên cứu}

Các hom thân tre Tầm vông được lấy từ các vườn của người dân tại thị xã Dĩ An, tỉnh Bình Dương đem về giâm ở vườn ươm tự tạo tại phường Tân Đông Hiệp, thị xã Dĩ An, tỉnh Bình Dương.

\subsection{Phương pháp nghiên cứu}

\subsubsection{Công tác lấy giống}

Chọn những cây tre có thời gian khoảng 1 năm tuổi để tiến hành lấy giống. Cây được chọn là những cây sinh trưởng bình thường, không sâu bệnh, đường kính cây từ $3,5 \mathrm{~cm}$ trở lên.

Sau khi đã đánh dấu xong những cây được chọn, tiến hành chặt hạ cây, bảo quản và chở ngay về vườn ươm. Khi về đến vườn ươm tiến hành cưa thành những hom giống để đem ươm. Hom giống được cưa sao cho mắt tre nằm giữa hom. Chiều dài của các hom giống khoảng $25 \mathrm{~cm}$.

\subsubsection{Chuẩn bị đất và giàn che}

Thực hiện làm đất trước khi giâm hom. Đất được cuốc tơi xốp, không còn cỏ dại. Trước khi giâm hom 1 tuần, tiến hành tưới thuốc để phòng trừ nấm. Làm thành từng luống (liếp) có chiều ngang khoảng $1-1,2 \mathrm{~m}$. Phía bên trên dùng lưới có độ tàn che $50 \%$ để che mát cho chồi tre sau khi mọc.

\subsubsection{Bố trí thí nghiệm}

Thí nghiệm về ảnh hưởng của chất điều hòa sinh trưởng: ảnh hưởng của chất điều hòa sinh trưởng đến khả năng nảy chồi và ra rễ của hom thân giống Tầm vông đem giâm được tiến hành nghiên cứu ở 3 loại chất với 4 nghiệm thức: Đối chứng (không xử lý chất ĐHST), NAA, IBA và HVP. HVP là sản phẩm thương mại của công ty cổ phần dịch vụ kỹ thuật nông nghiệp TP.HCM với thành phần $\mathrm{N}-\mathrm{P}_{2} \mathrm{O}_{5}-\mathrm{K}_{2} \mathrm{O}$ là $20-20-15$. Các chất điều hòa sinh trưởng được xử lý ở nồng độ 150 ppm trong thời gian ngâm là 120 phút. Thí nghiệm được bố trí theo kiểu hoàn toàn ngẫu nhiên (CRD) 1 nhân tố với 3 lần lặp, mỗi nghiệm thức gồm 36 hom.

Thí nghiệm về ảnh hưởng của vị trí lấy hom: ảnh hưởng của vị trí lấy hom trên cây mẹ đến khả năng nảy chồi và ra rễ của hom thân giống Tầm vông đem giâm được nghiên cứu ở 3 vị trí (gọi là 3 nghiệm thức): Các hom tre nằm gần gốc (V1), các hom tre nằm giữa thân $(\mathrm{V} 2)$ và các hom tre nằm ở gần ngọn (V3). Tất cả các hom tre ở cả 3 vị trí được xử lý chất điều hòa sinh trưởng NAA với nồng độ 200 ppm và thời gian ngâm là 120 phút. Nồng độ và thời gian được tiến hành ở thí nghiệm này dựa trên kết quả tốt nhất của thí nghiệm về ảnh hưởng của nồng độ và thời gian xử lý chất điều hòa sinh trưởng NAA. Thí nghiệm được bố trí theo kiểu hoàn toàn ngẫu nhiên (CRD) 1 yếu tố với 3 lần lặp, mỗi nghiệm thức gồm 36 hom.

Nhằm giúp cho hom giống được giâm có điều kiện thuận lợi hơn cho việc nảy chồi và ra rễ, thành phần giá thể ngoài đất gieo ươm tại vườn ườm còn được bổ sung thêm $5 \%$ phân chuồng hoai và $1 \%$ phân super lân.

\subsubsection{Giâm hom}

Hom sau khi đã ngâm chất điều hòa sinh trưởng, được đem ngay ra vườn để giâm. Để tiến hành giâm, dùng cuốc rạch thành từng rãnh nhỏ sâu khoảng $10 \mathrm{~cm}$. Bỏ hom xuống rãnh theo chiều nằm ngang, dùng tay ấn nhẹ để hom được nén chặt. Sau đó tiến hành lấp đất lại.

Thời gian giâm hom ở cả hai thí nghiệm được thực hiện vào mùa xuân (tháng 3 - 4 trong năm).

\subsubsection{Thu thập và xử lý số liệu}

Các chỉ tiêu nghiên cứu được thu thập như sau:

Tỷ lệ hom nảy chồi (\%): Xác định sau 21 ngày sau khi giâm.

Số lượng chồi(măng) trên mỗi hom: Xác định sau 21 ngày sau khi giâm.

Tỷ lệ hom ra rễ (\%), số lượng rễ trung bình trên hom(rễ/hom) và chiều dài của rễ $(\mathrm{cm})$ : Xác định sau 75 ngày sau khi giâm.

Đường kính chân măng $(\mathrm{mm})$ và chiều cao của cây măng $(\mathrm{cm})$ sau khi định hình thành cây khí sinh hoàn chỉnh. Xác định sau 75 ngày sau khi giâm.

Số liệu được xử lý theo phương pháp thống kê ứng dụng trong lâm nghiệp với sự trợ giúp của các phần mềm chuyên dụng trên máy vi tính: Micosoft Excel 2010 và Statgraphics Centurion XV.I.

Kết quả thí nghiệm được tiến hành phân tích phương sai (ANOVA) và phân hạng (bằng trắc 
nghiệm LSD) để so sánh giữa các nghiệm thức thí nghiệm bằng các chỉ tiêu đo đếm ở trên.

\section{Kết Quả và Thảo Luận}

3.1. Ảnh hưởng của loại chất điều hòa sinh trưởng đến tỷ lệ nảy chồi và số lượng chồi trên mỗi hom của hom thân tre Tầm vong Nam Bộ

Kết quả phân tích thống kê cho thấy loại chất điều hòa sinh trưởng có ảnh hưởng rất rõ rệt đến tỷ lệ nảy chồi của hom tre $(P<0,01$; Bảng 1$)$. Bảng phân hạng được chia ra làm 2 nhóm (các nghiệm thức nằm cùng 1 nhóm thì sự sai khác tỷ lệ nảy chồi giữa chúng là không có ý nghĩa) với giá trị từ thấp tới cao. Nhóm thứ nhất là nghiệm thức đối chứng, nhóm thứ 2 là 3 chất điều hòa sinh trưởng. Kết quả này cho thấy để giâm hom thân tre Tầm vông Nam Bộ đạt tỷ lệ nảy chồi cao thì cần phải xử lý các chất điều hòa sinh trưởng và theo thứ tự ưu tiên là NAA, IBA và cuối cùng là HVP. Kết quả phân tích thống kê cho thấy loại chất điều hòa sinh trưởng ảnh hưởng không rõ rệt đến tỷ lệ nảy chồi của hom tre $(P>0,05)$. Bảng phân hạng được phân ra chỉ 1 nhóm có cùng mức ý nghĩa giống nhau về tỷ lệ nảy chồi. Do không có sự khác biệt về số lượng chồi trên mỗi hom khi xử lý các chất điều hòa sinh trưởng khác nhau là do đặc tính sinh học của loài tre này, số lượng chồi đã được định hình trong mỗi mắt (đốt) tre trước khi giâm hom.

\section{2. Ảnh hưởng của loại chất điều hòa sinh trưởng đến đường kính chân măng và chiều cao măng (chồi) của hom thân tre Tầm vông Nam Bộ}

Kết quả phân tích thống kê cho thấy 3 loại chất NAA, IBA và HVP ảnh hưởng không rõ rệt đến đường kính của chân măng hom thân tre Tầm vông Nam Bộ $(P>0,05$; Bảng 2$)$. Đường kính chân măng sau khi phân hạng được chia làm 2 nhóm, nhóm thứ nhất là nghiệm thức đối chứng và 2 chất IBA và $\mathrm{HVP}$, nhóm thứ 2 là 3 chất NAA, IBA và HVP. Trong đó, NAA là chất điều hòa sinh trưởng cho đường kính chân măng lớn hơn 2 chất còn lại.

Kết quả phân tích thống kê cho thấy giữa 3 loại chất điều hòa sinh trưởng được xử lý và đối chứng có ảnh hưởng rất rõ rệt đến chiều cao của cây măng $(P<0,01)$. Kết quả phân hạng cho thấy chiều cao của cây măng sau khi xử lý 3 chất điều hòa sinh trưởng được chia thành 3 nhóm có giá trị từ thấp đến cao. Nhóm có giá trị cao nhất gồm 2 chất điều hòa sinh trưởng lần lượt là HVP và NAA.

Kết quả trên cũng phù hợp với nhận định của Ngo $(1999,2003)$ đường kính của măng và số đốt măng được quyết định từ trong hom tre, măng càng to thì số đốt càng nhiều. Các thay đổi của điều kiện môi trường chỉ làm thay đổi chủ yếu chiều dài của các giống tre.

3.3. Ảnh hưởng của loại chất điều hòa sinh trưởng đến tỷ lệ ra rễ, số lượng rễ và chiều dài rễ trung bình của hom thân tre Tầm vông Nam Bộ

Kết quả phân tích thống kê cho thấy, loại chất điều hòa sinh trưởng có ảnh hưởng rất rõ rệt đến tỷ lệ ra rễ của hom thân tre Tầm vông Nam Bộ $(P<0,01$; Bảng 3$)$. Tỷ lệ ra rễ sau khi phân hạng được chia thành 3 nhóm có giá trị từ thấp đến cao, nhóm có giá trị cao nhất gồm 2 chất điều hòa sinh trưởng lần lượt là NAA và IBA. Tương tự, chất điều hòa sinh trưởng có ảnh hưởng rất rõ rệt đến số lượng rễ trung bình trên mỗi hom thân tre Tầm vông Nam Bộ $(P<0,01)$. Bảng phân hạng được chia ra làm 3 nhóm có cùng mức ý nghĩa giống nhau về số lượng rễ trung bình với giá trị từ thấp tới cao. Nhóm thứ nhất là nghiệm thức đối chứng, nhóm thứ 2 là hai chất HVP và IBA, nhóm thứ 3 là hai chất IBA và NAA. Kết quả này cho thấy để giâm hom thân tre Tầm vông Nam Bộ đạt được số lượng rễ trung bình cao thì cần phải xử lý các chất điều hòa sinh trưởng và theo thứ tự ưu tiên là NAA, IBA.

Kết quả phân tích thống kê cũng cho thấy, 3 loại chất điều hòa sinh trưởng ảnh hưởng rất rõ rệt đến chiều dài rễ trung bình của cây hom thân tre Tầm vông Nam Bộ $(P<0,01)$. Bảng phân hạng được phân ra làm 2 nhóm có cùng mức ý nghĩa giống nhau về chiều dài rễ trung bình với giá trị từ thấp tới cao. Nhóm thứ nhất là nghiệm thức đối chứng, nhóm thứ 2 là 3 chất điều hòa sinh trưởng.

Kết quả nghiên cứu về tỷ lệ ra rễ và số lượng rễ trung bình của hom thân tre Tầm vông có phần tương tự với kết quả nghiên cứu của Sanjay \& ctv. (2004), NAA là chất chất điều hòa sinh trưởng cho kết quả tốt nhất đến khả năng ra rễ của loài Dendrocalamus asper, kế đến là IBA. 
Bảng 1. Tỷ lệ nảy chồi và số lượng chồi trên mỗi hôm của hom thân tre Tầm vong Nam Bộ sau khi xử lý các chất điều hòa sinh trưởng

\begin{tabular}{lcc}
\hline Loại chất điều hòa sinh trưởng & Tỷ lệ chồi $(\%)$ & Số chồi trên mỗi hom (Chồi/hom) \\
\hline Đối chứng & $51,87 \pm 1,87^{\mathrm{a}}$ & $3,20 \pm 0,16^{\mathrm{a}}$ \\
HVP $(150 \mathrm{ppm})$ & $86,10 \pm 1,60^{\mathrm{b}}$ & $3,38 \pm 0,11^{\mathrm{a}}$ \\
IBA $(150 \mathrm{ppm})$ & $87,03 \pm 2,45^{\mathrm{b}}$ & $3,36 \pm 0,11^{\mathrm{a}}$ \\
NAA $(150 \mathrm{ppm})$ & $91,67 \pm 1,60^{\mathrm{b}}$ & $3,39 \pm 0,12^{\mathrm{a}}$ \\
\hline$P$ & 0,00 & 0,18 \\
\hline a-b Các trị số có cùng ký tự đi kèm trong cùng một nhóm giá trị trung bình khác biệt không có ý nghĩa về mặt
\end{tabular}
thống kê $(P<0,05)$.

Bảng 2. Đường kính chân măng và chiều cao măng (chồi) của hom thân tre Tầm vong Nam Bộ sau khi xử lý các chất điều hòa sinh trưởng

\begin{tabular}{lcc}
\hline Loại chất điều hòa sinh trưởng & Đường kính chân măng $(\mathrm{cm})$ & Chiều cao măng $(\mathrm{cm})$ \\
\hline Đối chứng & $0,75 \pm 0,01^{\mathrm{a}}$ & $21,50 \pm 2,5^{\mathrm{a}}$ \\
HVP $(150 \mathrm{ppm})$ & $0,78 \pm 0,01^{\mathrm{ab}}$ & $51,19 \pm 1,08^{\mathrm{c}}$ \\
IBA $(150 \mathrm{ppm})$ & $0,78 \pm 0,01^{\mathrm{ab}}$ & $44,47 \pm 1,10^{\mathrm{b}}$ \\
NAA $(150 \mathrm{ppm})$ & $0,79 \pm 0,02^{\mathrm{b}}$ & $48,49 \pm 1,09^{\mathrm{bc}}$ \\
\hline$P$ & 0,15 & 0,00 \\
\hline a-c Các tri số có cùng ký tự đi kèm trong cùng một nhóm giá trị trung bình khác biệt không có ý nghĩa về mặt \\
thống kê $(P<0,05)$.
\end{tabular}

Bảng 3. Tỷ lệ ra rễ, số lượng rễ và chiều dài rễ trung bình của hom thân tre Tầm vong Nam Bộ sau khi xử lý các chất điều hòa sinh trưởng

\begin{tabular}{lccc}
\hline $\begin{array}{c}\text { Loại chất điều hòa } \\
\text { sinh trưởng }\end{array}$ & $\begin{array}{c}\text { Tỹ lệ ra rễ } \\
(\%)\end{array}$ & $\begin{array}{c}\text { Số lượng rễ trung bình } \\
(\text { rễ } / \text { hom })\end{array}$ & $\begin{array}{c}\text { Chiều dài rễ trung bình } \\
(\mathrm{cm})\end{array}$ \\
\hline Đối chứng & $1,85 \pm 0,09^{\mathrm{a}}$ & $5,00 \pm 0,1^{\mathrm{a}}$ & $4,90 \pm 0,04^{\mathrm{a}}$ \\
HVP $(150 \mathrm{ppm})$ & $80,56 \pm 1,60^{\mathrm{b}}$ & $7,06 \pm 0,36^{\mathrm{b}}$ & $8,71 \pm 0,09^{\mathrm{b}}$ \\
IBA $(150 \mathrm{ppm})$ & $82,41^{\mathrm{b}} \pm 2,45^{\mathrm{c}}$ & $7,40 \pm 0,35^{\mathrm{bc}}$ & $8,53 \pm 0,09^{\mathrm{b}}$ \\
NAA $(150 \mathrm{ppm})$ & $87,04 \pm 1,85^{\mathrm{c}}$ & $8,50 \pm 0,39^{\mathrm{c}}$ & $8,50 \pm 0,13^{\mathrm{b}}$ \\
\hline$P$ & 0,000 & 0,004 & 0,000 \\
\hline
\end{tabular}

${ }^{\mathrm{a}-\mathrm{c}}$ Các trị số có cùng ký tự đi kèm trong cùng một nhóm giá trị trung bình khác biệt không có ý nghĩa về mặt thống kê $(P<0,05)$.

\section{4. Ảnh hưởng của vị trí lấy hom đến tỷ lệ nảy chồi và số lượng chồi trên mỗi hom của thân tre Tầm vong Nam Bộ}

Kết quả phân tích thống kê cho thấy, vị trí lấy hom ảnh hưởng không rõ rệt đến tỷ lệ nảy chồi của hom thân tre Tầm vông Nam Bộ $(P>0,05$; Bảng 4). Bảng phân hạng cho thấy tỷ lệ nảy chồi ở 3 vị trí lấy hom cùng nằm trong một nhóm, nghĩa là không có sự sai khác về tỷ lệ nảy chồi giữa 3 vị trí lấy hom.

Kết quả phân tích thống kê cho thấy, giưa 3 vị trí lấy hom có ảnh hưởng rất rõ rệt đến số lượng chồi trung bình trên mỗi hom $(P<0,01)$. Bảng phân hạng cũng cho thấy số lượng chồi trung bình của hom lấy ở 3 vị trí khác nhau được chia thành 3 nhóm có giá trị từ thấp đến cao. Số lượng chồi trung bình của hom lấy ở vị trí gần ngọn là cao
Bảng 4. Tỷ lệ nảy chồi (\%) và số lượng chồi trên mỗi hom của hom thân tre Tầm vông Nam Bộ ở các vị trí lấy hom khác nhau

\begin{tabular}{ccc}
\hline $\begin{array}{c}\text { Vị trí } \\
\text { lấy hom }\end{array}$ & $\begin{array}{c}\text { Tỷ lệ nảy } \\
\text { chồi }(\%)\end{array}$ & $\begin{array}{c}\text { Số chồi trên mỗi } \\
\text { hom (Chồi/hom) }\end{array}$ \\
\hline V1 & $90,73 \pm 2,45^{\mathrm{a}}$ & $2,28 \pm 0,07^{\mathrm{a}}$ \\
$\mathrm{V} 2$ & $92,60 \pm 0,93^{\mathrm{a}}$ & $3,49 \pm 0,09^{\mathrm{b}}$ \\
$\mathrm{V} 3$ & $95,33 \pm 0,93^{\mathrm{a}}$ & $4,24 \pm 0,08^{\mathrm{c}}$ \\
\hline$P$ & 0,20 & 0,00 \\
\hline
\end{tabular}

$\overline{{ }^{\mathrm{a}-\mathrm{c}} \text { Các trị số có cùng ký tự đi kèm trong cùng một nhóm }}$ giá trị trung bình khác biệt không có ý nghĩa về mặt thống kê $(P<0,05)$.

nhất (4,24 chồi/hom), kế đến là vị trí giữa thân (3,49 chồi/hom) và thấp nhất là vị trí gần gốc (2,28 chồi/hom). Kết quả này có phần đối lập đối với kết quả nghiên cứu của Reena \& ctv. (2016) 
về vị trí lấy hom cho 2 loài Bambusa balcoo và Dendrocalamus asper (số lượng chồi của hom lấy ở vị trí gần gốc cao hơn hẳn so với vị trí giữa thân và thấp nhất là ở vị trí gần ngọn).

\section{5. Ảnh hưởng của vị trí lấy hom đến đường kính chân măng và chiều cao măng (chồi) của hom thân tre Tầm vông Nam Bộ}

Kết quả phân tích thống kê cho thấy, hom thân tre Tầm vông Nam Bộ lấy ở 3 vị trí khác nhau ảnh hưởng rất rõ rệt đến đường kính của chân măng $(P<0,01 ;$ Bảng 5$)$. Đường kính chân măng sau khi phân hạng được chia làm 3 nhóm có cùng mức ý nghĩa giống nhau có giá trị từ thấp đến cao, nhóm thứ nhất là hom lấy ở vị trí gần ngọn, nhóm thứ 2 là hom lấy ở vị trí gần gốc và nhóm thứ 3 là hom lấy ở vị trí giữa thân. Như vậy, khi lấy hom ở vị trí giữa thân sẽ cho đường kính chân măng là lớn nhất, kế đến là gần gốc và nhỏ nhất là gần ngọn.

Bảng 5. Đường kính chân măng và chiều cao măng (chồi) của hom thân tre Tầm vông Nam Bộ ở các vị trí lấy hom khác nhau

\begin{tabular}{ccc}
\hline $\begin{array}{c}\text { Vị trí } \\
\text { lây hom }\end{array}$ & $\begin{array}{c}\text { Đường kính chân } \\
\text { măng }(\mathrm{cm})\end{array}$ & $\begin{array}{c}\text { Chiều cao } \\
\text { măng }(\mathrm{cm})\end{array}$ \\
\hline V1 & $0,77 \pm 0,02^{\mathrm{b}}$ & $46,38 \pm 0,79^{\mathrm{a}}$ \\
V2 & $0,84 \pm 0,01^{\mathrm{c}}$ & $50,37 \pm 0,99^{\mathrm{b}}$ \\
V3 & $0,73 \pm 0,01^{\mathrm{a}}$ & $46,83 \pm 1,33^{\mathrm{a}}$ \\
\hline$P$ & 0,20 & 0,02 \\
\hline
\end{tabular}

${ }^{\mathrm{a}-\mathrm{c}}$ Các trị số có cùng ký tự đi kèm trong cùng một nhóm giá trị trung bình khác biệt không có ý nghĩa về mặt thống kê $(P<0,05)$.

Chiều cao trung bình của cây măng (chồi) của hom lấy ở vị trí giữa thân là lớn nhất $(50,37 \mathrm{~cm})$, kế đến là vị trí gần ngọn $(46,83 \mathrm{~cm})$ và thấp nhất là vị trí gần gốc $(46,38 \mathrm{~cm})$. Kết quả phân tích thống kê cho thấy, giữa 3 vị trí lấy hom có ảnh hưởng rõ rệt đến chiều cao của cây măng $(P<$ $0,05)$. Qua bảng phân hạng cho thấy chiều cao của cây măng được phân thành 2 nhóm có giá trị từ thấp đến cao, nhóm thứ nhất là hom lấy ở vị trí gần gốc và gần ngọn, nhóm thứ 2 là hom lấy ở vị trí giữa thân.

Kết quả trên cho thấy, khi lấy hom ở các vị trí khác nhau trên thân cây tre Tầm vông sẽ cho đường kính chân măng và chiều cao của măng là khác nhau (Hình 1). Kết quả này có phần khác biệt đối với kết quả nghiên cứu của Reena \& ctv. (2016) cho 2 loài Bambusa balcoo và Dendrocalamus asper (chiều dài của măng lấy ở vị trí gần gốc cao hẳn so với vị trí giữa thân và thấp nhất là ở vị trí gần ngọn).

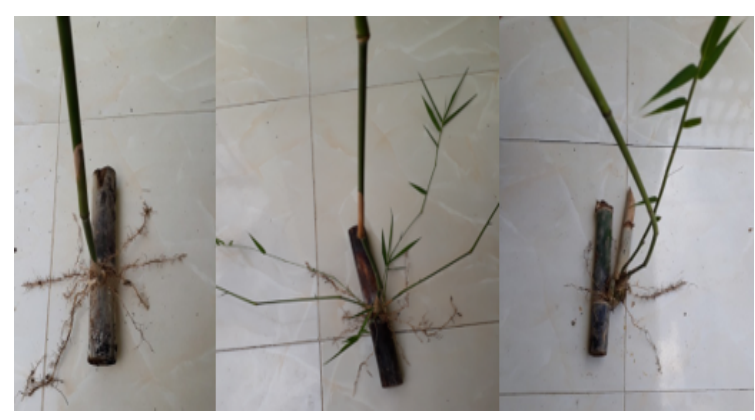

Hình 1. Hom tre ở 3 vị trí khác nhau ra rễ sau 75 ngày tuổi.

(a): Vị trí gần gốc (V1), (b): Vị trí giữa thân (V2), (c): Vị trí gần ngọn (V3).

\section{6. Ảnh hưởng của vị trí lấy hom đến tỷ lệ ra rễ, số lượng rễ và chiều dài rễ trung bình của hom thân tre Tầm vông Nam Bộ}

Kết quả phân tích thống kê cho thấy, vị trí lấy hom có ảnh hưởng rõ rệt đến tỷ lệ ra rễ của hom thân tre Tầm vông Nam Bộ $(P<0,05$; Bảng 6$)$. Tỷ lệ ra rễ sau khi phân hạng được chia thành 2 nhóm có giá trị từ thấp đến cao, nhóm có giá trị cao hơn gồm 2 vị trí lấy hom lần lượt là giữa thân và gần gốc. Kết quả này cũng phù hợp với nghiên cứu của Sanjay \& ctv. (2004), những hom giâm ở gần gốc cho tỷ lệ ra rễ tốt hơn những hom giâm ở phần ngọn.

Số lượng rễ trung bình cao nhất khi lấy hom ở vị trí gần gốc (9,7 rễ/hom), kế đến là vị trí giữa thân $(8,6$ rễ/hom) và thấp nhất là vị trí gần ngọn (7,13 rê/hom). Kết quả phân tích thống kê cũng cho thấy vị trí lấy hom có ảnh hưởng rất rõ rệt đến số lượng rễ trung bình trên mỗi hom thân tre Tầm vông Nam Bộ $(P<0,01)$. Bảng phân hạng được chia ra làm 3 nhóm số lượng rễ trung bình với giá trị từ thấp tới cao. Nhóm thứ nhất là vị trí lấy hom gần ngọn, nhóm thứ 2 là vị trí lấy hom giữa thân và nhóm thứ 3 là vị trí lấy hom gần gốc. Kết quả thí nghiệm cho thấy, khi hom càng gần gốc thì số lượng rễ ra được trên mỗi hom sẽ cao hơn khi hom gần về phía ngọn của cây tre Tầm vông Nam Bộ. Kết quả này tương tự với nghiên cứu của Reena \& ctv. (2016) cho 2 loài Bambusa balcooa và Dendrocalamus asper. Khi hom càng gần gốc thì số lượng rễ trên mỗi hom sẽ cao hơn, hom càng gần ngọn thì số lượng rễ của hom khi giâm sẽ giảm dần. 
Bảng 6. Tỷ lệ ra rễ, số lượng rễ và chiều dài rễ trung bình của hom thân tre Tầm vông Nam Bộ ở các vị trí lấy hom khác nhau

\begin{tabular}{cccc}
\hline $\begin{array}{c}\text { Vị trí } \\
\text { lấy hom }\end{array}$ & $\begin{array}{c}\text { Tỷ lệ ra rễ } \\
(\%)\end{array}$ & $\begin{array}{c}\text { Số lượng rễ trung bình } \\
(\text { rễ } / \text { hom })\end{array}$ & $\begin{array}{c}\text { Chiều dài rễ trung bình } \\
(\mathrm{cm})\end{array}$ \\
\hline V1 & $87,96 \pm 0,93^{\mathrm{b}}$ & $9,70 \pm 0,39^{\mathrm{c}}$ & $8,41 \pm 0,09^{\mathrm{b}}$ \\
V2 & $89,82 \pm 0,93^{\mathrm{b}}$ & $8,60 \pm 0,33^{\mathrm{b}}$ & $8,71 \pm 0,08^{\mathrm{c}}$ \\
V3 & $82,41 \pm 2,45^{\mathrm{a}}$ & $7,13 \pm 0,31^{\mathrm{a}}$ & $8,07 \pm 0,08^{\mathrm{a}}$ \\
\hline$P$ & 0,017 & 0,000 & 0,003 \\
\hline a-c Các trì số có cùng ký tự đi kèm trong cùng một nhóm giá trị trung bình khác biệt không có ý
\end{tabular}

Kết quả phân tích thống kê cho thấy, vị trí lấy hom ảnh hưởng rất rõ rệt đến chiều dài rễ trung bình của cây hom thân tre Tầm vông Nam Bộ $(\mathrm{P}<0,01)$. Bảng phân hạng được phân ra làm 3 nhóm có cùng mức ý nghĩa giống nhau về chiều dài rễ trung bình với giá trị từ thấp tới cao. Nhóm cao nhất là vị trí lấy hom ở giữa thân với chiều dài rễ trung bình $8,71 \mathrm{~cm}$. Kết quả này có phần khác so với nghiên cứu của Reena \& ctv. (2016) cho 2 loài Bambusa balcooa và Dendrocalamus asper. Khi hom càng gần gốc thì chiều dài rễ của hom sẽ cao hơn, hom càng gần ngọn thì chiều dài rễ của hom khi giâm sẽ giảm dần.

\section{Kết Luận}

Khi giâm hom thân tre Tầm vông Nam bộ, 2 chất NAA và IBA cho tỷ lệ nảy chồi, số lượng chồi trên mỗi hom, đường kính chân măng, tỷ lệ ra rễ và số lượng rễ trung bình luôn nằm trong nhóm cao nhất của bảng phân hạng. Trong đó, NAA cho kết quả tốt hơn so với IBA. Bên cạnh đó, HVP là chất điều hòa sinh trưởng có ảnh hưởng đến chiều cao măng và chiều dài rễ lớn hơn 2 chất còn lại.

Hom tre Tầm vông Nam Bộ lấy ở vị trí giữa thân (V2) cho đường kính chân măng, chiều cao trung bình của măng, tỷ lệ ra rễ và chiều dài rễ trung bình là lớn nhất, kế đến là vị trí gần gốc (V1) và thấp nhất là vị trí gần ngọn (V3). Tuy nhiên, số lượng rễ trung bình cao nhất khi lấy hom ở vị trí gần gốc (V1).

\section{Tài Liệu Tham Khảo (References)}

Ngo, D. Q. (2003). Bamboos (planting and use). Nghe An, Vietnam: Nghe An Publishing House.

Ngo, D. Q. (1999). Planting techniques for some multipurpose woody plants. Ha Noi, Vietnam: Ethnic Minorities' Culture Publishing House.

Nguyen, N. H. (2005). Bamboos in Vietnam. Ha Noi, Vietnam: Agricultural Publishing House.

Reena, J., Tewari, S. K., Kaushal, R., Deepa, R., Supriya, \& Pradeep M. (2016). Effect of position of culm cut tings on the growth parameters of bamboos. Progressive Research - An International Journal 11 (SpecialVII), 5000-5002.

Sanjay, S., Pramod, K., \& Ansari, S. A. (2004). A simple method for large-scale propagation of Dendrocalamus asper. Scientia Horticulturae 100(1-4), 251-255. 